\title{
Le fructose-2,6-bisphosphate chez les mammifères
}

Le fructose-2,6-bisphosphate, activateur de la phosphofructokinase 1 et inhibiteur de la fructose-1,6-bisphosphatase, joue un rôle pivot dans la régulation de l'équilibre entre la glycolyse et la néoglucogenèse hépatiques. Présent dans de nombreuses cellules animales et végétales, il peut être impliqué non seulement dans le contrôle de la glycolyse, mais aussi dans d'autres types de régulation encore mal connus.

Louis Hue Mark H. Rider

\section{ADRESSE}

L. Hue, M.H. Rider : unité hormones et métabolisme, université catholique de Louvain (UCL 75-29) et international institute of cellular and molecular pathology, 75, avenue 'étude du mécanisme d'action du glucagon sur la néoglucogenèse hépatique a mené à la découverte du fructose-2,6-bisphosphate (F-2,6-BP) [1, 2] dont la structure est développée sur la figure 1. Le F-2,6-BP est un puissant activateur allostérique de la 6-phosphofructo-1-kinase (PFK-1), une des enzymes clés de la glycolyse ; il est également un inhibiteur de la fructose-1,6-bisphosphatase (FBPase-1), une enzyme spécifique de la néoglucogenèse. Le F-2,6-BP est actif à des concentrations micromolaires qui correspondent aux taux normalement trouvés dans les cellules. Le F-2,6-BP est un composé largement répandu dans le monde animal et végétal ; il n'a cependant pas encore été détecté chez les procaryotes. Étant donné ce caractère ubiquiste, le F-2,6-BP apparaît donc comme un candidat privilégié pour le contrôle de la glycolyse. Nous détaillerons plus loin les tissus et les conditions dans lesquels le F-2,6-BP contrôle effectivement le flux glycolytique. Nous verrons également que les propriétés des enzymes catalysant la synthèse et la dégradation de cette molécule differrent considérablement d'un tissu à l'autre. Dans la plupart des manuels de biochimie, on considère que le rôle principal de la glycolyse se limite à la récupération de l'énergie d'oxydation du glucose sous forme d'adénosine triphosphate (ATP). C'est de fait un rôle important de la glycolyse, mais ce n'est pas le seul, loin s'en faut. En effet, le lactate et le pyruvate, produits de la glycolyse, sont des précurseurs pour la synthèse de novo des acides gras, du cholestérol et de certains acides aminés. En ce sens, la glycolyse peut participer à des processus biosynthétiques. Nous verrons que le mode d'action du F-2,6-BP sur la PFK-1, le rend particulièrement bien adapté pour contrôler cette fonction anabolique de la glycolyse.

Plusieurs articles de revue ont déjà été consacrés au F-2,6BP [3-9]. Le lecteur pourra y trouver les références aux travaux originaux et des détails concernant, entre autres, les effets du F-2,6-BP sur la PFK-1 et la FBPase-1, la régulation de sa synthèse et de sa dégradation, et son rôle dans le contrôle de la glycolyse.

\section{PFK-1 et FBPase-1}

Le fructose-2,6-bisphosphate stimule la phosphofructokinase et inhibe la fructose-1,6-bisphosphatase. Il existe plusieurs isozymes de la PFK-1 qui présentent cependant des propriétés cinéti- 


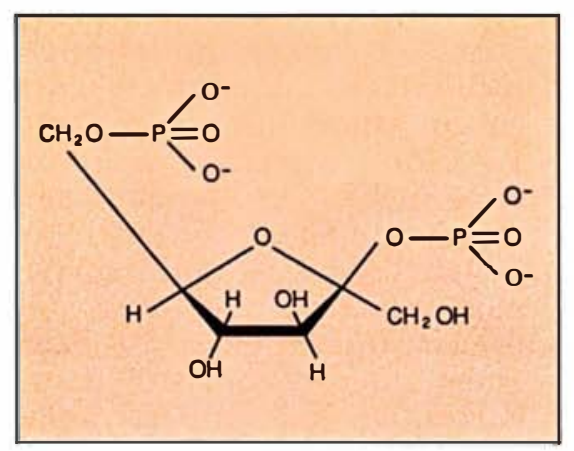

Figure 1. Structure du $\beta$-D-fructose2,6-bisphosphate.

Figure 2. Contrôle de la glycolyse et de la néoglucogenèse hépatiques par lo fructose-2,6-bisphosphato. Les traits pleins symbolisent les réactions chimiques alors que les lignes discontinues symbolisent les influences régulatrices. FBPase-1 = fructose-1,6-bisphosphatase ; FBPase-2 = fructose-2,6-bisphosphatase ; PFK-1 = 6-phosphofructo-1kinase ; PFK-2 = 6-phosphofructo-2kinase $; \mathrm{Pi}=$ phosphate inorganique ; ATP $=$ adénosine triphosphate $; A D P$ = adénosine diphosphate.

ques assez semblables. L'ATP qui est un des substrats de la réaction se comporte comme un effecteur allostérique négatif. Il induit une coopérativité pour le fructose-6phosphate, l'autre substrat. Cette inhibition par l'ATP peut être levée en augmentant la concentration en effecteurs positifs. Parmi ceux-ci, on trouve le fructose-6phosphate et surtout l'adénosine monophosphate (AMP) et le F-2,6-BP. Ces differents effecteurs agissent en synergie. Les propriétés cinétiques de la PFK-1 du foie sont telles que, dans les conditions physiologiques, l'enzyme est inactive sauf en présence de concentrations physiologiques de F-2,6-BP, ce qui supprime l'inhibition par l'ATP et permet ainsi à la glycolyse de se faire. Par conséquent, dans le foie et peut-être dans d'autres tissus, le F-2,6-BP est le candidat de choix pour stimuler la PFK-1 lorsque la fonction anabolique de la glycolyse est

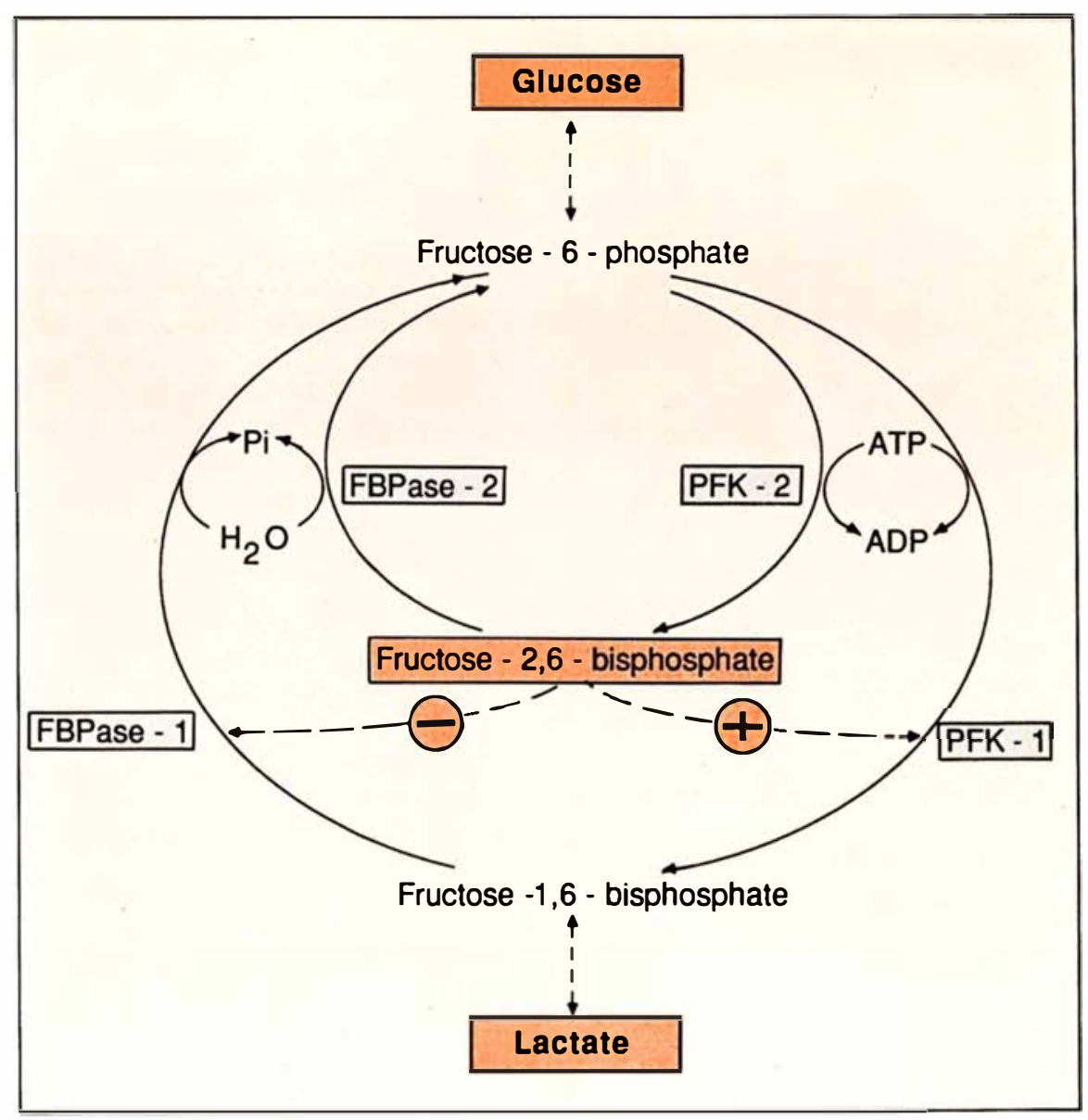

prépondérante. En revanche, lorsque la glycolyse est la seule source d'ATP, comme par exemple en anoxie, il est probable que ce sont d'autres effecteurs de la PFK-1, tels que les nucléotides de l'adénine, qui jouent le rôle le plus important. Toutes les PFK-1 des tissus de mammiferes étudiés jusqu'à présent sont stimulées par le F-2,6-BP.

Le F-2,6-BP est un inhibiteur de la FBPase-1. Il agit en synergie avec l'AMP et l'inhibition est plus importante à faibles concentrations qu'à fortes concentrations en substrat. A la différence de la PFK-1 qui est présente dans tous les tissus, la FBPase-1 ne se trouve en quantités appréciables que dans les tissus capables de réaliser la néoglucogenèse, c'est-à-dire dans le foie et le cortex rénal. Donc, dans le foie, le F-2,6-BP est un élément clé du contrôle de la glycolyse et de la néoglucogenèse (figure 2). Toute augmentation de sa concentration entraînera une stimulation de la PFK-1 (glycolyse) et une inhibition de la FBPase-1 (néoglucogenèse). Cependant la FBPase-1 est pres de 10 fois moins sensible au F-2,6-BP que ne l'est la PFK-1. Il s'ensuit que ces deux enzymes peuvent agir simultanément permettant ainsi l'existence d'un cycle futile entre le fructose-6phosphate et le fructose-1,6-bisphosphate. De fait, ce cycle n'est pas détectable lorsque la concentration hépatique en $\mathrm{F}-2,6$ - $\mathrm{BP}$ est très faible (à jeun), alors qu'il le devient dans le foie d'animaux nourris (voir plus loin).

A côté de ces effets sur la PFK-1 et la FBPase-1, le F-2,6-BP peut également stimuler une phosphofructokinase de plantes qui utilise le pyrophosphate comme donneur de phosphate. L'enzyme extraite de tubercules de pommes de terre, qui est particulièrement sensible au F-2,6-BP, est couramment uti- 
lisée pour le dosage de microquantités $\left(10^{-10} \mathrm{~g}\right)$ de $\mathrm{F}-2,6-\mathrm{BP}$.

\section{F-2,6-BP : synthèse et dégradation}

La synthèse et la dégradation du fructose-2,6-bisphosphate sont catalysées par une même enzyme. Les propriétés du système enzymatique du foie seront décrites brièvement avant d'être comparées à celles des autres tissus (figure 3 et Tableau I). La synthèse de F-2,6-BP à partir de fructose-6-phosphate et d ATP est catalysée par la 6-phosphofructo-2-kinase (PFK-2). Dans les conditions normales, l'enzyme n'est pas saturée par son substrat, le fructose-6-phosphate. L'hydrolyse du F-2,6-BP en fructose-6phosphate et $\mathrm{Pi}^{*}$ est catalysée par une phosphatase spécifique, la fructose-2,6-bisphosphatase (FBPase-2). L'enzyme est normalement saturée par son substrat le F-2,6-BP ; cependant, le fructose6-phosphate, un produit de la réaction, inhibe la FBPase-2 avec un $\mathrm{Ki}^{* *}$ égal ou supérieur à la concentration cellulaire en fructose-6-phosphate. Par conséquent, tout changement dans la concentration en fructose-6-phosphate devrait provoquer des changements dans le même sens de la concentration en F-2,6-BP. On a de fait pu montrer qu'il existe une relation directe entre ces deux paramètres. Parmi les autres modulateurs de l'activité de ces deux enzymes, il faut retenir le $\mathrm{Pi}$, un stimulateur des deux enzymes, le citrate, un inhibiteur de la PFK-2, et le glycérol-3-phosphate qui inhibe la PFK-2 mais stimule la FBPase-2.

1. Van Schaftingen E, Hue L, Hers HG. Control of the fructose-6-phosphate/fructose1,6-bisphosphate cycle in isolated hepatocytes by glucose and glucagon. Biochem J 1980 ; 192 : 887-95.

2. Van Schaftingen E, Hue L, Hers HG. Fructose-2,6-bisphosphate, the probable structure of the glucose - and glucagon - sensitive stimulator of phosphofructokinase. Biochem J $1980 ; 192: 897-901$

3. Hers HG, Van Schaftingen E. Fructose-2,6-bisphosphate two years after its discovery. Biochem J $1982 ; 206: 1-12$.

4. Uyeda K, Furuya E, Richards CS, Yokoama M. Fructose-2,6- $\mathrm{P}_{2}$ chemistry and biological function. Mol Cell Biochem 1982; 48 : 97-120.

5. Pilkis SJ, El-Maghrabi MR, McGrane $M$, Pilkis J, Fox E, Claus TH. Fructose-2,6-bisphosphate : A mediator of hormone action at the fructose-6-phosphate/fructose-1,6-bisphosphate substrate cycle. Mol Cell Endocrinol 1982 ; $25: 245-66$

6. Hers HG, Hue L. Gluconeogenesis and related aspects of glycolysis. Annu Rev Biochem $1983 ; 52: 617-53$.

7. Claus TH, El-Maghrabi MR, Regen DM et al. The role of fructose-2,6-bisphosphate in the regulation of carbohydrate metabolism. Cur Top Cell Regul 1984; 23 : 57-86.

8. Van Schaftingen E. Fructose-2,6-bisphosphate Adv Enzymol 1987 ; 59 : 315-95.

9. Hue L, Rider M. Role of fructose-2,6-bisphosphate in the control of glycolysis in mammalian tissues. Biochem $J 1987 ; 245: 313-24$.

- Phosphate inorganique.

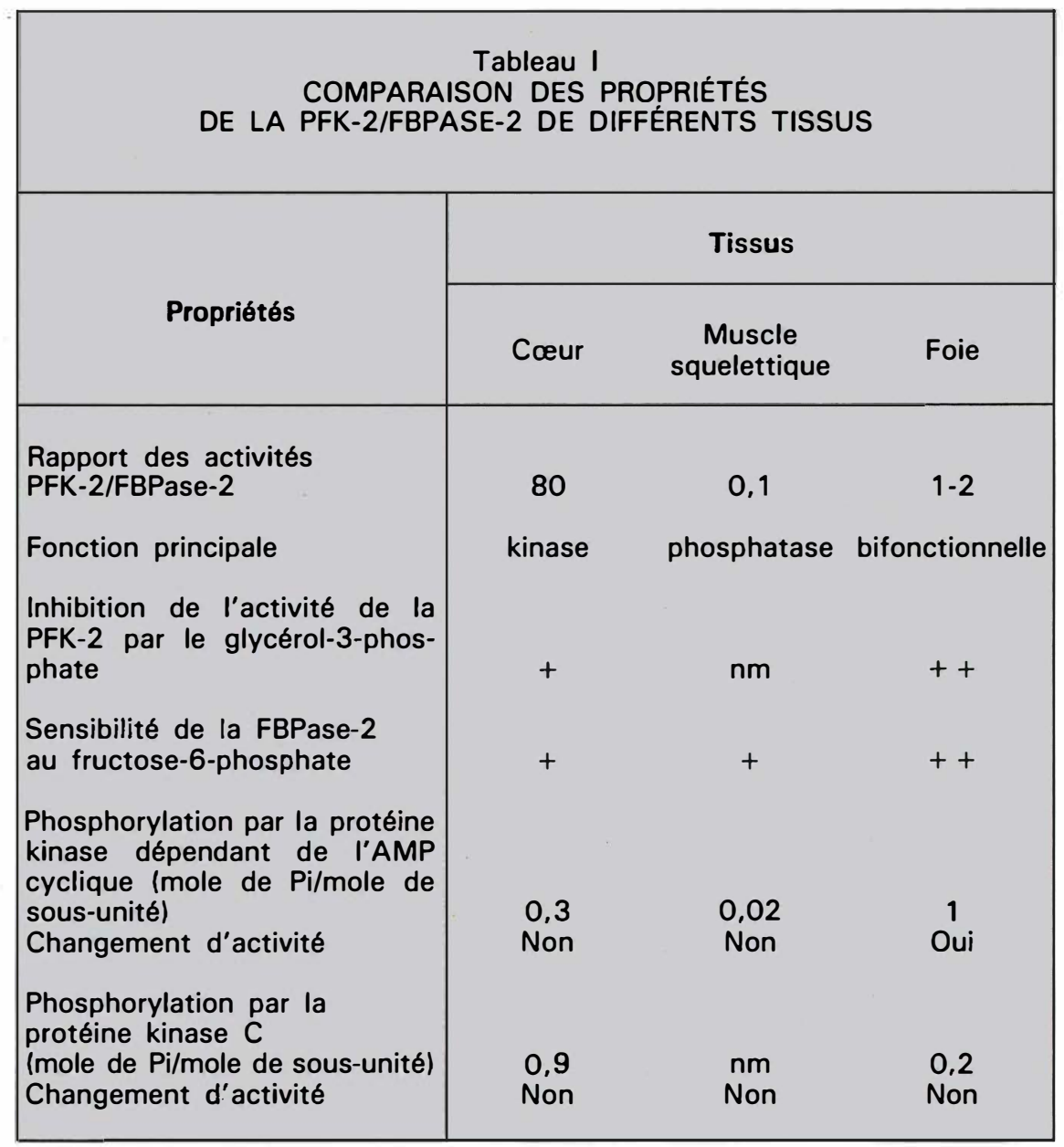

$\mathrm{nm}=$ non mesure $; \mathrm{Pi}=$ phosphate inorganique $;$ PFK-2 $=6$-phosphofructo-2kinase; FPBase-2 = fructose-2,6-bisphosphatase. 


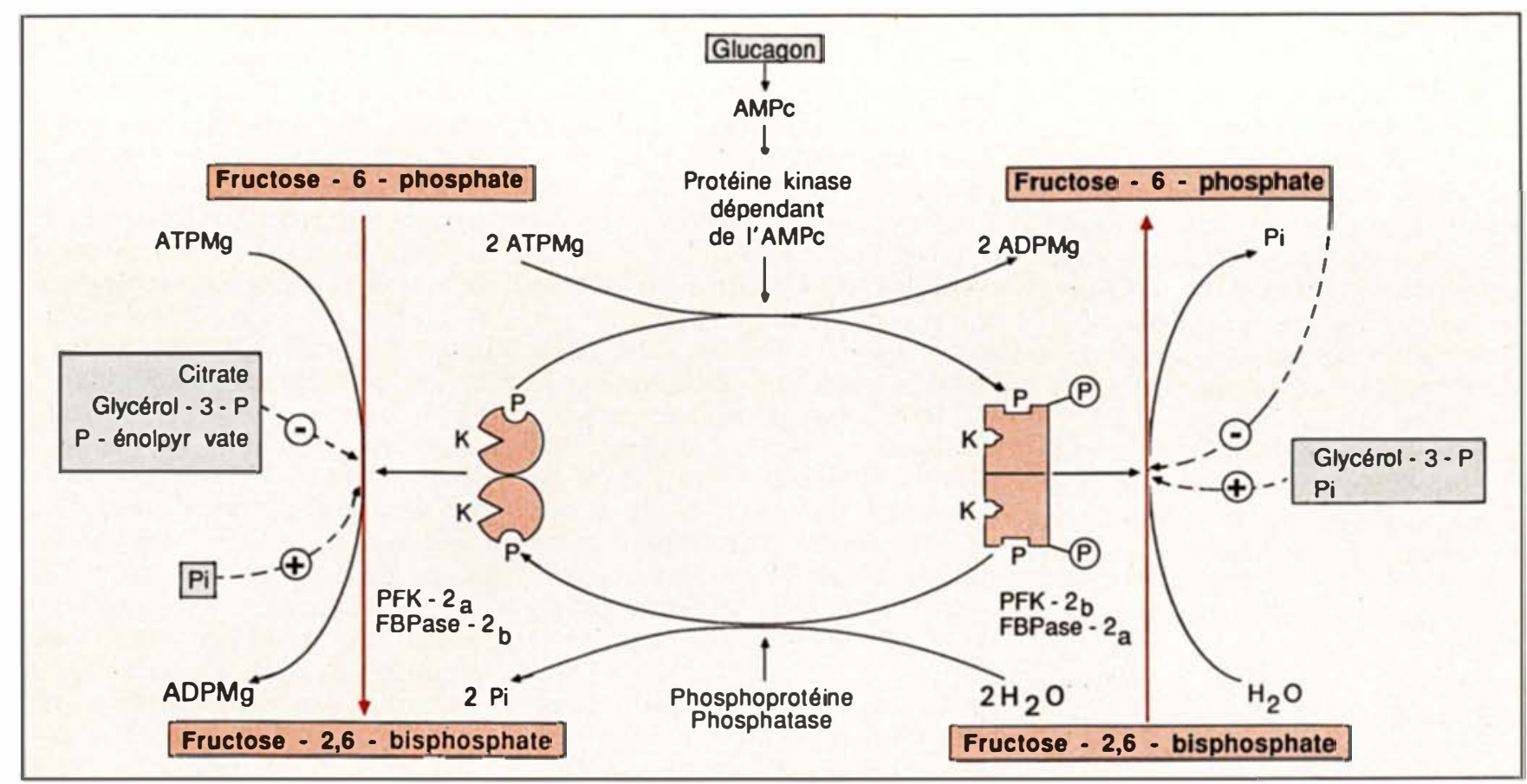

Figure 3. Contrôle de la synthèse et de la dégradation du fructose-2,6-bisphosphate dans le foie. Les symboles utilisés (traits pleins et discontinus) sont les mêmes que dans la figure 2. FBPase-2 = fructose-2,6-bisphosphatase ( $a=$ active, $b=$ inactive) $;(P)=$ site "phosphatase "; PFK-2 = 6-phosphofructo-2-kinase $(a=$ active, $b=$ inactive) ; $K=$ site "kinase "; $P=$ résidu sérine phosphorylé ; $P i=$ phosphate inorganique ; $A M P c=$ adénosine monophosphate cyclique $; A T P=$ adénosine triphosphate $; M g=$ magnésium $;$ glycérol-3-P $=$ glycérol-3-phosphate ; $P$ énolpyruvate = phosphoénolpyruvate ; $A D P=$ adénosine diphosphate.

Plusieurs arguments indiquent qu'une seule et même protéine est porteuse de la PFK-2 et de la FBPase-2. Cette protéine bifonctionnelle, la PFK-2/FBPase-2, est composée de deux sous-unités identiques qui, chacune, portent deux sites catalytiques distincts. Dans le foie, cette protéine bifonctionnelle est un substrat de la protéine kinase dépendant de l'AMP cyclique qui inactive la PFK-2 et active la FBPase-2 par phosphorylation.

L'étude des propriétés des PFK-2/FBPase- 2 de cœur et de muscle squelettique a montré qu'il existe différents isozymes de la PFK-2/FBPase-2. Ces isozymes se distinguent par leurs propriétés cinétiques, par le rapport des activités kinasique et phosphatasique et par leur réponse au traitement par les protéines kinases. On a également pu montrer que l'enzyme du cœur n'était pas reconnue par des anticorps dirigés contre l'enzyme de foie. Alors que l'enzyme du foie contient presque autant d'activité kinasique que phosphatasique, l'enzyme du cœur contient au moins 10 fois plus de kinase que de phosphatase tandis que l'enzyme du muscle est plus riche en phosphatase. Jusqu'à présent, la régulation de l'activité de la PFK-2/FBPase-2 par la protéine kinase dépendant de l'AMP cyclique n'a été observée qu'avec l'enzyme du foie. En revanche, l'enzyme du cœur (mais pas celle du foie) peut être phosphorylée par la protéine kinase $C$; il s'agit d'une phosphorylation "silencieuse ", c'est-à-dire qui ne s'accompagne pas de changement d'activité.

Il est intéressant de noter que la PFK-2 de levure est également un substrat de la protéine kinase dépendant de l'AMP cyclique. Dans ce cas, la phosphorylation résulte en une activation de la PFK-2 contrairement au foie où une inactivation est observée.

\section{Glycolyse et F-2,6-BP}

Relation entre la glycolyse et le fructose-2,6-bisphosphate dans le foie. Dans le foie, on peut schématiquement envisager trois états métaboliques qui se caractérisent par des flux glycolytiques et néoglucogéniques différents. La première situation correspond à l'état de jeûne et au traitement par le glucagon. Dans ce cas, la néoglucogenèse est active tandis que la glycolyse est bloquée. La concentration en F-2,6-BP est très basse (inférieure à $10^{-6} \mathrm{M}$ ) ce qui s'explique par l'inactivation de la PFK-2 et l'activation de la FBPase-2 résultant de la phosphorylation par la protéine kinase dépendant de l'AMP cyclique. Dans ces conditions, la glycolyse est bloquée non seulement du fait de l'inhibition de la PFK-1 par la baisse du F-2,6-BP, mais également parce que la pyruvate kinase est inactivée par le glucagon, en vertu d'une phosphorylation qui procède du même mécanisme que celui décrit ci-dessus.

Une baisse de la concentration en F-2,6-BP peut aussi être observée dans le foie en régénération, et dans des hépatocytes incubés en présence d'éthanol, d'adénosine ou d'acides gras. Pour expliquer la baisse en F-2,6-BP dans ces 
conditions, on peut invoquer des changements de concentrations d'AMP cyclique et de régulateurs de la PFK-2/FBPase-2 tels que le glycérol-3-phosphate et le citrate. La seconde situation correspond à l'abondance de glucose, comme par exemple après un repas riche en glucides. Dans ces conditions, le foie consomme du glucose dont une partie est stockée sous forme de glycogène et le reste est transformé en lactate et acides gras. Le glucose est par lui-même capable d'exercer un contrôle à la fois sur la glycolyse et sur la synthèse du glycogène. Il est à noter que dans ces conditions de pléthore, la néoglucogenèse n'est pas entièrement inhibée et qu'il existe donc un cycle futile entre le fructose-6phosphate et le fructose-1,6-bisphosphate. La stimulation de la glycolyse s'explique par une augmentation de la concentration en F-2,6-BP, elle-même résultant de l'augmentation de la concentration des hexoses 6-phosphate, induite par le glucose. La stimulation de la synthèse de glycogène provient de l'activation de la glycogène synthase faisant suite à l'inactivation de la glycogène phosphorylase causée par le glucose lui-même. Il semble que la sensibilité au glucose de la glycolyse et de la synthèse de glycogène ne soit pas la même. Chez l'animal à jeun, l'activation de la glycogène synthase requiert moins de glucose que la stimulation de la glycolyse ; l'inverse est vrai chez l'animal nourri. La signification physiologique de cette observation est que, après administration de glucose à l'animal à jeun, les réserves de glycogène sont d'abord reconstituées et ce n'est que lorsqu'on apporte un excès de glucose que la néoglucogenèse est ralentie et que du lactate est produit.

On peut observer une augmentation de F-2,6-BP hépatique non seulement après administration de glucose à l'animal normal mais aussi au cours du jeûne chez des animaux génétiquement obèses. Cette glycolyse élevée pourrait nant une lipogenèse active pendant le jeûne.

Dans des hépatocytes de rats normaux, une relation directe entre la concentration en F-2,6-BP et la production nette de lactate ne s'observe qu'à des concentrations relativement élevées de F-2,6-BP (supérieures à $5 \mathrm{nmol} / \mathrm{g}$ ). Cette absence de relation s'explique par le fait qu'il existe un cycle futile entre le fructose-6-phosphate et le fructose-1,6-bisphosphate et que, en dessous de $5 \times 10^{-6} \mathrm{M}$, plus de $90 \%$ du F-2,6-BP est lié aux protéines. Il est à noter que ce dernier phénomène ne s'observe que dans le foie. Une augmentation de la concentration en F-2,6-BP peut également s'obtenir dans des hépatocytes isolés de rats nourris lorsque ces cellules sont traitées par la vasopressine ou par des agents $\alpha$-adrénergiques. Ces substances stimulent la dégradation du glycogène par un mécanisme indépendant de l'AMP cyclique. La glycogénolyse mène à une augmentation de la concentration en fructose-6-phosphate et en F-2,6-BP car, contrairement au glucagon, ces agents ne stimulent pas l'adénylate cyclase et donc ne provoquent pas d'inactivation de la PFK-2 ni d'activation de la FBPase-2.

La troisième situation métabolique du foie correspond à celle où la glycolyse est stimulée de façon maximale par l'anoxie. Cette situation extrême ne s'observe probablement jamais dans les conditions physiologiques mais on peut l'obtenir facilement in vitro. Dans ces conditions, la concentration en F-2,6-BP s'abaisse ou reste basse et on ne peut donc pas attribuer au F-2,6-BP un rôle dans la stimulation de la glycolyse en anaérobiose. Contrairement au foie, le muscle squelettique en contraction peut passer par des épisodes hypoxiques voire même anoxiques. Dans ces conditions, comme dans le foie anoxique, il semble que la stimulation maximale de la glycolyse ne soit pas due au F-2,6-BP.

Relation entre la glycolyse et le fructose-2,6-bisphosphate dans les tissus extra-hépatiques.
D'une façon générale, la glycolyse peut être influencée soit par la disponibilité et la concentration en glucose, son substrat, soit par la demande énergétique, par exemple en anaérobiose, soit enfin par certaines hormones et facteurs de croissance. La question est donc de savoir si l'on peut attribuer au F-2,6-BP un rôle de régulateur dans ces différentes conditions.

En ce qui concerne la régulation par le glucose, on a montré qu'il existe un parallélisme assez strict entre la glycolyse et la concentration en F-2,6-BP dans tous les tissus étudiés, sauf dans les astrocytes en culture, les spermatozoïdes et les globules rouges. Il est intéressant de noter que ces cellules dépendent de la glycolyse pour leur approvisionnement en ATP. Ainsi qu'il a déjà été mentionné plus haut, il n'y a pas de parallélisme entre glycolyse et F-2,6-BP au cours de l'anoxie. D'autre part, dans les tissus qui peuvent oxyder d'autres substrats que le glucose (par exemple des acides gras et des corps cétoniques), une diminution de la concentration en F-2,6-BP pourrait signaler la présence de ces substrats non glycolytiques et ainsi contribuer à l'épargne glucidique. Dans le muscle squelettique et dans le cœur, le F-2,6-BP est également impliqué dans la stimulation de la glycolyse par l'adrénaline et l'insuline. De plus, on a montré que dans le cœur, l'insuline activait la PFK-2 ; le mécanisme de cette activation est cependant inconnu. Il faut enfin mentionner que l'on connaît très peu de choses concernant le rôle du F-2,6-BP dans le cerveau, tissu dans lequel l'apport énergétique dépend essentiellement de la glycolyse.

\section{Cellules tumorales et $\mathrm{F}-2,6-B P$}

Le fructose-2,6-bisphosphate dans les cellules tumorales et les cellules en prolifération. Ces cellules se caractérisent par un flux glycolytique élevé qu'elles conservent même en aérobiose. Le mécanisme biochimique de ce phénomène connu depuis plus de 
60 ans n'est cependant pas encore entièrement élucidé. Cette différence par rapport aux cellules normales pourrait résulter, au moins en partie, de changements qualitatifs et quantitatifs de l'activité de certaines enzymes et transporteurs. Des travaux récents ont indiqué que, en plus des différences déjà observées, le F-2,6-BP pourrait jouer un rôle important dans le maintien d'un flux glycolytique anormalement élevé dans ces cellules. En effet, dans certaines lignées de cellules cancéreuses, la concentration en F-2,6-BP est plus grande que dans le tissu normal correspondant. De plus, l'addition de facteurs de croissance ou de certains esters de phorbol à des cultures de fibroblastes d'embryon de poulet entraîne une stimulation de la glycolyse et une augmentation concomitante de la concentration en F-2,6-BP, de même qu'une activation de la PFK-2. Une situation identique est obtenue lorsque ces fibroblastes sont transformés par des rétrovirus porteurs des oncogènes $v$-src ou v-fps. Les modifications observées en présence du virus de sarcome de Rous dépendent de l'activité tyrosine kinase de $\mathrm{p} 60 \mathrm{v}$-src, la protéine codée par l'oncogène. La comparaison des effets de $\mathrm{v}$-src et de ceux des esters de phorbol sur le $\mathrm{F}-2,6-\mathrm{BP}$ suggère que ces effets pourraient résulter de la stimulation d'une étape commune contrôlant l'activation de la PFK-2 ; cette étape serait également sous le contrôle de la protéine kinase C. Le mécanisme exact est encore inconnu.

Les changements du système F-2,6-BP/PFK-2 que l'on observe dans les fibroblastes transformés ne sont pas les seuls déterminants du phénotype glycolytique. En effet, le transport de glucose est également stimulé et la contribution relative de ces deux phénomènes au contrôle général de la glycolyse est difficile à évaluer. Il n'en reste pas moins qu'une caractéristique des cellules en prolifération est leur concentration très élevée en F-2,6-BP. En fait il s'agit des concentrations les plus élevées que l'on ait jamais trouvées. Ces concentrations sont au moins 10 fois plus grandes que celles requises pour stimuler la PFK-1 et on est tenté de se demander si le F-2,6-BP ne pourrait pas influencer d'autres voies métaboliques que la glycolyse. On ignore si ces concentrations importantes en F-2,6-BP sont requises pour maintenir les cellules en phase de prolifération. Il est intéressant de noter que des changements semblables dans la concentration de F-2,6-BP ont été observés au cours du réveil de la dormance chez les plantes et dans certaines spores.

\section{AMP cyclique et F-2, 6-BP}

La comparaison des modes d'action du F-2,6-BP et de l'AMP cyclique, fait ressortir des différences fondamentales entre ces deux signaux. Le F-2,6-BP se comporte comme un intégrateur qui concentre sur un seul point de contrôle, la PFK-1, une série d'informations provenant de différents métabolismes. L'AMP cyclique au contraire amplifie l'information en la faisant passer d'une molécule, l'hormone, à différentes voies métaboliques. Ceci est dû aux propriétés remarquables de la protéine kinase qui est une enzyme à substrats multiples, alors que la PFK-2/FBPase-2 est une enzyme multi-modulée qui peut donc agir comme intégrateur métabolique. De plus, l'AMP cyclique est capable d'agir sur le F-2,6-BP, alors que l'inverse n'est pas vrai. Ceci suggère qu'il existe une hiérarchie entre ces deux signaux et que le contrôle rapide par les hormones dépendant de l'AMP cyclique se superpose à un contrôle plus lent qui, lui, dépend de la disponibilité des substrats énergétiques

\section{TIRÉS A PART}

L. Hue : unité hormones et métabolisme, université catholique de Louvain (UCL 75-29) et international institute of cellular and molecular pathology, 75, avenue Hippocrate, 1200 Bruxelles, Belgique.

\section{Summary}

Fructose-2,6-bisphosphate $(\mathrm{F}-2,6-\mathrm{BP})$ is a potent stimulator of 6-phosphofructo-1. kinase, a key glycolytic enzyme, and an inhibitor of fructose-1,6bisphosphatase, a gluconeogenic enzyme. F-2,6-BP is synthesized from fructose-6-phosphate and ATP by 6 -phosphofructo-2kinase (PFK-2) and converted back to fructose-6-phosphate by fructose-2,6-bisphosphatase (FBPase-2). Both activities are borne by a single bifunctional protein composed of two subunits, each of them bearing two distinct catalytic sites. The liver bifunctional enzyme is a substrate of the cyclic AMP dependent protein kinase which inactivates PFK-2 and activates FBPase-2. Experimental data suggest the existence data suggest the existence of several PFK-2 isoenzymes which differ by their kinetic properties, their FBPase-2 content and their ability to become phosphorylated. Although F-2,6-BP is present in all tissues studied so far, it can not be regarded as the regulator of glycolysis under all conditions. F-2, 6-BP probably plays no role in tissues that depend exclusively on glycolysis for their energy supply. In tissues that can use alternative fuels such as fatty acids, a fall in F-2,6-BP concentration could signal the presence of nonglycolytic substrates and so contribute to the glucose-sparing effect of these substrates. In skeletal muscle and heart, F-2,6-BP is also involved in the control of glycolysis by adrenaline and insulin. In liver, it is a major control element for switching from glycolysis to gluconeogenesis and could be important in the control of substrate provision for lipogenesis. Finally, $\mathrm{F}-2,6-\mathrm{BP}$ is probably essential for maintaining the high aerobic rate of glycolysis which is a characteristic feature of fastgrowing célls. 